\title{
Scheduling Algorithms for Procrastinators
}

\author{
Michael A. Bender* Raphaël Clifford ${ }^{\dagger} \quad$ Kostas Tsichlas $^{\ddagger}$
}

\begin{abstract}
If once a man indulges himself in murder, very soon he comes to think little of robbing; and from robbing he comes next to drinking and Sabbath-breaking, and from that to incivility and procrastination.

— Thomas de Quincey
\end{abstract}

\begin{abstract}
This paper presents scheduling algorithms for procrastinators, where the speed that a procrastinator executes a job increases as the due date approaches. We give optimal off-line scheduling policies for linearly increasing speed functions. We then explain the computational/numerical issues involved in implementing this policy. We next explore the online setting, showing that there exist adversaries that force any online scheduling policy to miss due dates. This impossibility result motivates the problem of minimizing the maximum interval stretch of any job; the interval stretch of a job is the job's flow time divided by the job's due date minus release time. We show that several common scheduling strategies, including the "hit-the-highest-nail" strategy beloved by procrastinators, have arbitrarily large maximum interval stretch. Then we give the "thrashing" scheduling policy and show that it is a $\Theta(1)$ approximation algorithm for the maximum interval stretch.
\end{abstract}

\section{Introduction}

We are writing this sentence two days before the deadline. Unfortunately that sentence (and this one) are among the first that we have written. How could we have delayed so much when we have known about this deadline for months? The purpose of this paper is to explain why we have waited until the last moment to write this paper.

In our explanation we model procrastination as a scheduling problem. We cannot use traditional scheduling algorithms to model our behavior because such algorithms do not take into account our (and humanity's) tendency to procrastinate. The advantages of procrastination are well documented: the closer to a deadline a task is executed, the less processing time the task appears to require. Hence, it is common for a person to delay executing some onerous job in order to spend as little time as possible working on it.

Regarding this paper, it will certainly be written quickly — it will have to be, since the deadline is near. Perhaps we will write faster under pressure because we will expend less time overanalyzing each design option. Other aspects of the paper may change because of this time pressure. In any case, the writing will proceed faster than if we had begun earlier.

Our scheduling problem for procrastinators is unusual in that the processing time of a job depends on the times when the job is run. We are given as input a set of jobs $\mathcal{J}=\{1,2, \ldots, n\}$. Each job $j$ has release time

\footnotetext{
*Department of Computer Science, Stony Brook University, Stony Brook, NY 11794-4400, USA. Email: benderecs. sunysb. edu. This research was supported in part by NSF Grants CCR-0208670, CCF-0621439/0621425, CCF-0540897/05414009, CCF-0634793/0632838, and CNS-0627645.

${ }^{\dagger}$ Department of Computer Science, University of Bristol, Merchant Venturers Building, Woodland Road, Bristol BS8 1UB, UK. Email: cliffordecs.bris.ac.uk.

${ }^{\ddagger}$ Computer Engineering and Informatics Department, University of Patras, 26500 Patras, Greece. Email: tsihlaseceid.upatras.gr.
} 
$r_{j}$, due date $d_{j}$, and work $w_{j}$; without loss of generality, we assume that the jobs are indexed by increasing release times. Preemption is allowed; that is, a running job can be interrupted and resumed later. The speed at which job $j$ is run depends on the times that $j$ is executed; the closer to the due date $d_{j}$, the faster $j$ can be executed. Specifically, speed function $f_{j}(t)$ indicates that at time $t$, job $j$ is executed with speed $f_{j}(t)$; thus, if $j$ is executed during time interval $[a, b]$, then $\int_{t=a}^{b} f_{j}(t) d t$ units of work of job $j$ complete.

Throughout most of the paper we focus on linear speed functions. We assume that when job $j$ first is released, it is executed with speed 0 . In accordance with this last assumption, when the call for papers first appeared, we snapped into action and accomplished nothing.

Despite our whimsical and self-referential style, we hope to emphasize that the scheduling problems on job streams with time-dependent processing times have mathematical subtlety as well as practical relevance. The time-dependent processing models in this paper may be useful for industry and sociology because they give better scheduling models of human behavior; no model can truly be accurate that does not account for people's ability to work faster under the temporary stress of deadlines. More generally, many common scheduling problems in both daily life and industry have tasks whose processing times are time-dependent. For example, an airplane that is late in arriving may have the boarding procedure expedited, a construction project that is behind may have more workers assigned to it, and a shipment that is late may be delivered faster by using an alternative, more expensive means of transportation. Indeed a major reason for the success of companies such as Fedex, UPS, and DHL is that the world is filled with scheduling problems executed by procrastinators.

\section{Related Work}

A number of other optimization problems have well studied time-dependent variants, including work on time-dependent shortest paths [25] and time-dependent flows [16, 17]. Some authors, typically in the operations-research community, have also worked on scheduling with time-dependent processing times (see, e.g., $[2,5,18,19])$, but for the offline and nonpremptive case. Of course, preemptive and online models are best for modeling the behavior of procrastinators, who tend to timeshare and thrash as the deadlines approach. Moreover, our introduction of preemptive scheduling with time-dependent processing times requires an entirely different model. Previous work has assumed that the processing time $p_{j}(t)$ for job $j$ is a function of the starting time $t$. We cannot have such a model in a preemptive case because the job may be executed during many different time intervals. This issue motivates our need for processor speeds: job $j$ is executed with speed $f_{j}(t)$ at time $t$; the processing time is the sum over all intervals when job $j$ is executed, and the integral of $f_{j}(t)$ over all times that the job is executed must equal the job's work. Curiously, if we analyze existing nonpreemptive models (e.g., linearly decreasing processing times) and analyze what processor speeds and total work must be to generate these processing times, then we can create instances where the processing speeds approach infinity; clearly such a model is unrealistic.

The most closely related work in the literature is on scheduling algorithms for minimizing power consumption and, in particular, on "speed scaling." See [1,6,7,10,27,28] for some recent results and [21] for an excellent survey. The idea of speed scaling is that the processing speed of a job is variable, but faster speeds consume more power. This ability to vary the speeds is reminiscent of the procrastinator who can run at unsustainable rates near the deadline. However, unlike in the speed-scaling model, the procrastinator has less freedom in choosing the processing speed; the processing speed is solely determined by the proximity to the deadline.

We note that there exist other scheduling papers where processors have different speeds, both for "related" processors [9, 11, 12] and for "unrelated" processors [13, 22, 24]. However, neither situation models procrastination scheduling (or speed scaling), where the processing speeds per job change over time.

There are other scheduling problems on how to schedule reluctant workers, such as the lazy bureaucrat problem $[3,4,20]$. However, the lazy bureaucrats in the scheduling problem are trying to accomplish as few 
of the jobs as possible, whereas the procrastinators in the current scheduling problem are trying to finish all of the jobs.

\section{Results}

In this paper we present the following results.

- Optimal offline scheduling - We first give optimal offline scheduling policies for the case where a scheduling instance has a feasible solution. We consider the case of linear speed functions, $f_{j}(t)=$ $m_{j}\left(t-r_{j}\right)$, for constant $m_{j} \geq 0$. (In the offline problem, the scheduler sees the entire problem instance before it has to begin scheduling.) Specifically, the policy gives the feasible solution in which the processors spend the minimum total time running. These results are consistent with a procrastinator who, after missing crucial deadlines, muses "if I could do it all over again...."

- Computational/numerical issues - We show that, curiously, despite a simple optimal scheduling policy, actually determining feasibility of the resulting schedule is not even known to be in NP. In particular, determining feasibility is hard because of the computational difficulties of summing square roots. We know of few scheduling problems where this intriguing issue arises.

- Online scheduling - We next turn to online scheduling. Not surprisingly, the feasibility problem is not achievable in an online setting. In particular, even if the online procrastinator has a feasible set of jobs, he/she may be forced to miss an arbitrarily large number of due dates.

- Online maximum interval stretch - A procrastinator may be forced to execute jobs beyond their due dates, that is, for some job $j$, the completion time $C_{j}$ may exceed the due date $d_{j}$. Generally speaking, if a procrastinator has a year to do a job $j$, and completes $j$ two weeks late, the situation is better than if the procrastinator has only one day to do $j$, but completes two weeks late. This observation motivates the notion of interval stretch, defined as the flow time (time the job spends in the system) divided by the job's interval. More formally, the interval stretch 1 of job $j$ is defined as $s_{j}=\left(C_{j}-r_{j}\right) /\left(d_{j}-r_{j}\right)$. We consider the optimization metric maximum interval stretch (abbreviated to max-stretch), $\max _{j} s_{j}$.

We study online scheduling of feasible scheduling instances. We explore traditional scheduling policies for the procrastinator, such as First-In-First-Out (FIFO), Shortest-Remaining-Processing-Time (SRPT), and earliest-due-date (EDD). We show, not surprisingly, that these policies do not perform well and can lead to unbounded max-stretch. A common scheduling policy among many procrastinators is "hit-the-highest-nail", that is, execute the task that most crucially requires attention, formally, Largest-Stretch-So-Far (LSSF). In LSSF we execute the job in the system that currently has the largest interval stretch. We prove, perhaps surprisingly, that LSSF can lead to arbitrarily large max-stretch. We conclude our exploration of max-stretch by exhibiting an online algorithm for the procrastinator, THRASHING, that yields $\Theta(1)$ max-stretch. This last result holds even when each job has a bound on its maximum execution speed.

\section{Offline Procrastination Scheduling}

In this section we consider the offline procrastination-scheduling problem. First, we give an optimal scheduling policy based on a simple priority rule. Then we show that it is computationally difficult to determine whether a scheduling instance is feasible, despite this priority rule. We focus on linear speed functions,

\footnotetext{
${ }^{1}$ This definition deviates from the standard notion of stretch where the flow time is divided by the total time the job has spent working [8]. However, it is appropriate here as jobs have due dates which can be missed and job speed is time-dependent.
} 


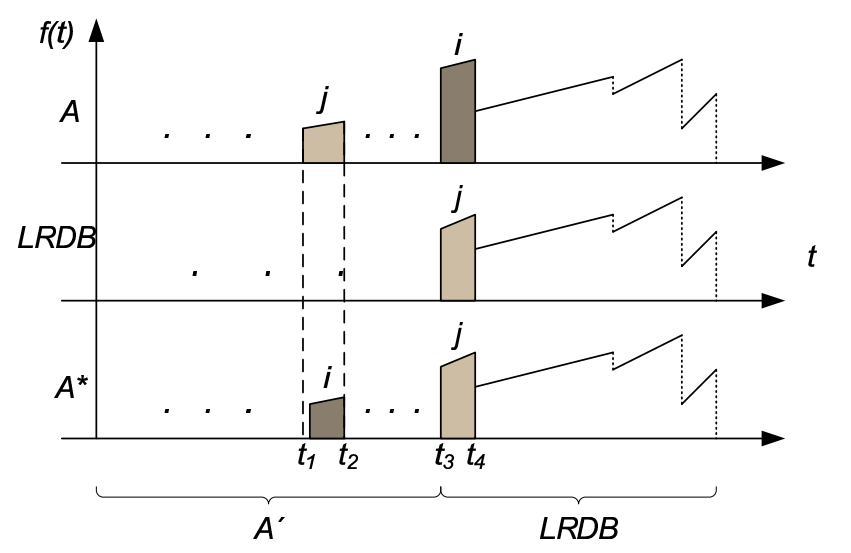

Figure 1: Schedule $A^{*}$ results from the merge of $A^{\prime}$ and LRTB. Schedule $A^{\prime}$ results from $A$ by exchanging jobs $j$ and $i$. The small gap after $t_{1}$ indicates that this exchange is more time efficient.

$f_{j}(t)=m_{j}\left(t-r_{j}\right)$. We will show that, without loss of generality, we can assume that all speed functions can have unit slope, i.e., that $m_{j}=1$.

\section{Optimal Offline Scheduling Policy}

We now give an optimal scheduling policy for the offline procrastination problem based on a simple priority rule.

We first define terms. We say that a schedule is feasible if all jobs complete within their intervals; we say that a feasible schedule is optimal if the total processing time is minimized. Observe that if an optimal schedule has no idle time then all feasible schedules are also optimal.

The optimal algorithm starts at the latest due date and works backwards in time, prioritizing jobs by the latest release time. Whenever a new job is encountered (at the job's due date) or a job completes, then the job in the system having the latest release time is serviced. Where two or more jobs have the same release time the scheduler chooses between them in an arbitrary but fixed way. We call this scheduling algorithm Latest Release Time Backwards (LRTB).

Observe that LRTB is the traditional Earliest Due Date (EDD) policy (see, e.g., [23]) when we reverse the flow of time so that release dates become due dates and due dates become release dates. In traditional scheduling, time can flow in either direction, so that both LRTB and EDD generate feasible schedules. In contrast, in the procrastination problem, EDD performs poorly; see Section 3 . The intuition of the algorithm is that it always tries to push the work of a job as near to its due date as possible in order to maximize the processing speed.

Observe that the job priorities depend only on the release times and not the slopes. This lack of dependence on the slopes should not be surprising because we can transform any scheduling instance into an instance having all unit slopes by rescaling each job $j$ 's work to be $w_{j}^{\prime}=w_{j} / m_{j}$. Alternatively, we could give all jobs unit maximum speeds, $f_{j}\left(d_{j}\right)=1$, by setting $m_{j}=1 /\left(d_{j}-r_{j}\right)$ and then rescaling the work. Consequently, in the rest of the paper, we assume that the job slopes are 1, unless otherwise stated.

In the following we prove that algorithm LRTB produces the optimal schedule.

Theorem 1 LRTB is an optimal algorithm for the procrastination scheduling problem. There is a unique optimal solution provided that the release time of the jobs are distinct.

Proof. The proof is by an exchange argument. We first assume that no two jobs have the same release time and then relax that assumption at the end. Suppose for the sake of contradiction that there exists an optimal 
schedule $A$ different from LRTB. Specifically, these schedules differ in the order of execution of two jobs with different release times. We perform a single exchange of work to yield another feasible schedule $A^{*}$ having smaller total processing time than $A$, thus obtaining a contradiction.

Consider the latest instant in time where LRTB differs from $A$ and call this time $t_{4}$. Consider an arbitrarily small interval $\left[t_{3}, t_{4}\right]$, when job $j$ runs in LRTB and job $i$ runs in $A$. See Figure 1 for a depiction of the setting. By the definition of LRTB, $i, j$, and $t_{4}, r_{i}<r_{j}$. Consider some earlier time interval $\left[t_{1}, t_{2}\right]$, i.e., $t_{2} \leq t_{3}$, when job $j$ runs in $A$. Define $t_{1}, t_{2}$, and $t_{3}$ so that the amount of work that can be executed on job $j$ is the same, that is,

$$
\int_{t=t_{1}}^{t_{2}} f_{j}(t) d t=\int_{t=t_{3}}^{t_{4}} f_{j}(t) d t
$$

Now we make a new schedule $A^{*}$ from $A$ by exchanging the work done during intervals $\left[t_{1}, t_{2}\right]$ to $\left[t_{3}, t_{4}\right]$. Specifically in $A^{*}$, job $j$ is run during $\left[t_{3}, t_{4}\right]$ and job $i$ is run during $\left[t_{1}, t_{2}\right]$. We know that this exchange is allowed because $d_{j}>t_{4}$ (from the LRTB and $A$ schedules) and because $r_{i}<r_{j} \leq t_{1}$ (from the $A$ schedule and because $r_{i}<r_{j}$ ). By the definition of the intervals, the same amount of work on $j$ can be done during each interval. Computing the area of the trapezoids defined by $f_{j}(t)$, we obtain

$$
\left(t_{4}-t_{3}\right)\left(\frac{t_{4}+t_{3}}{2}-r_{j}\right) m_{j}=\left(t_{2}-t_{1}\right)\left(\frac{t_{2}+t_{1}}{2}-r_{j}\right) m_{j}
$$

meaning that

$$
\left(t_{4}^{2}-t_{3}^{2}\right) / 2-r_{j}\left(t_{4}-t_{3}\right)=\left(t_{2}^{2}-t_{1}^{2}\right) / 2-r_{j}\left(t_{2}-t_{1}\right) .
$$

Observe that $t_{4}-t_{3}<t_{2}-t_{1}$ because the speed that $j$ is executed during $\left[t_{3}, t_{4}\right]$ is greater than during $\left[t_{1}, t_{2}\right]$.

The amount of work on job $i$ that needs to be exchanged from $\left[t_{3}, t_{4}\right]$ to $\left[t_{1}, t_{2}\right]$ is $\left(t_{4}^{2}-t_{3}^{2}\right) / 2-\left(t_{4}-t_{3}\right) r_{i}$. But since $r_{i}<r_{j}$ and $t_{4}-t_{3}<t_{2}-t_{1}$,

$$
\left(r_{j}-r_{i}\right)\left(t_{4}-t_{3}\right)<\left(r_{j}-r_{i}\right)\left(t_{2}-t_{1}\right)
$$

From (1) and (2), we obtain the inequality

$$
\left(t_{4}^{2}-t_{3}^{2}\right) / 2-r_{i}\left(t_{4}-t_{3}\right)<\left(t_{2}^{2}-t_{1}^{2}\right) / 2-r_{i}\left(t_{2}-t_{1}\right)
$$

and therefore interval $\left[t_{1}, t_{2}\right]$ is big enough to execute all of the work on job $i$ and still leave some idle time. Hence, schedule $A^{*}$ is feasible and spends a smaller amount of time working. This gives us our contradiction.

We now explain the case where two jobs 1 and 2 have the same release time. Assume that job 1 is scheduled to execute some work in the time interval $\left[t_{1}, t_{2}\right]$ and job 2 is scheduled to execute some work in the interval $\left[t_{3}, t_{4}\right]$. If we exchange the work for jobs 1 and 2 , the relationship between the new time intervals and the old is expressed by the simple equation $t_{4}^{2}-t_{3}^{2}=t_{2}^{2}-t_{1}^{2}$. Therefore the total time to execute both jobs remains the same after exchange. As a result, the order in which these jobs are executed does not affect the total processing time, and so LRTB is an optimal algorithm no matter what the tie-breaking rule is. This completes the proof.

\section{Determining Feasibility May Not Be in NP}

One of the remarkable features of the procrastination problem is that, despite having the simple optimal scheduling policy LRTB, it is unclear whether determining the feasibility of a scheduling instance is even in $\mathrm{NP}$, even for linear speed functions. 
The difficulty is numerical. Calculating the actual processing time of the job $j$ given a starting or ending time $t$ and speed function $f_{j}(t)=t-r_{j}$ requires computing square roots. Determining the feasibility of the schedule therefore requires computing sums of square roots and their relationship to an integer, and this problem appears to be numerically difficult.

The basic sum-of-square-roots problem is to determine whether

$$
\sum_{i=1}^{m} \sqrt{x_{i}} \geq I
$$

for some $x_{i}, I \in \mathbb{Z}(1 \leq i \leq m)$. Because there is no known polynomial-time algorithm for deciding the sum-of-square-roots problem, basic computational-geometry problems such as Euclidean TSP or Euclidean shortest paths are not known to be in NP. See the Open Problems Project [14, Problem 33] (originally from [26]) and the Geometry Junkyard [15] for nice discussions of the sum-of-square-roots problem.

We establish the difficulty of procrastination scheduling by providing a reduction from any instance of the sum-of-square-roots problem. To derive the cleanest reduction, we allow the existence of nonlazy jobs, i.e., jobs that are always executed at the same speed, i.e., having slope 0 . (It is likely that a reduction can be made to work using no nonlazy jobs, but at the cost of additional complications.)

Theorem 2 The procrastination scheduling problem is not decidable in polynomial time unless the sum-ofsquare-roots problem is decidable in polynomial time. The procrastination scheduling problem is not in NP unless the sum-of-square-roots problem is also in NP.

Proof. We reduce the sum-of-square-roots problem to the procrastination scheduling problem. Given integers $x_{1}, \ldots, x_{n-1}$ and $I$, we will create a procrastination-scheduling problem with $n$ jobs. The procrastination scheduling problem will be feasible if and only if $\sum_{i=1}^{n-1} \sqrt{x_{i}} \geq I$.

We first give the structure of the scheduling instance and then determine the release times, deadlines, and work for each job. In our scheduling instance, jobs $1 \ldots n-1$ have nonoverlapping intervals, so that $r_{1}=0$, and the due date of one job is the release date of the next: $r_{i+1}=d_{i}(i=1, \ldots, n-2)$. The speed functions have slope 1 . Job $n$ is nonlazy. We place this job's interval so that it overlaps with the intervals of all other jobs, i.e., $r_{n}=r_{1}$ and $d_{n}=d_{n-1}$.

We now specify jobs $1, \ldots, n-1$. For job $i$, we choose interval length $\ell_{i}\left(=d_{i}-r_{i}\right)$ and work $w_{i}$ to be positive integers such that $\ell_{i}^{2}-2 w_{i}=x_{i}$; many choices of $\ell_{i}$ and $w_{i}$ will work. It suffices to choose positive integers $\ell_{i}$ and $w_{i}$ such that $0<\ell_{i}^{2}-2 w_{i}<\ell_{i}$. For example, by choosing $\ell_{i}=x_{i}+2$ and $w_{i}=\left(x_{i}^{2}+3 x_{i}+4\right) / 2$, all conditions are fulfilled. Note that $x_{i}^{2}+3 x_{i}+4$ is always an even number for $x_{i}>0$ and thus $w_{i}$ is an integer.

Each job $i(i=1, \ldots, n-1)$ runs fastest when pushed to the right side of its interval. We show that such a job runs in time $t_{i}=\ell_{i}-\sqrt{\ell_{i}^{2}-2 w_{i}}$. To establish this running time, we set up and solve a quadratic equation. By simple geometry, we have the following relationship between running time $t_{i}$ and work $w_{i}$ :

$$
w_{i}=t_{i}\left(\ell_{i}-t_{i} / 2\right) .
$$

This quadratic equation has two roots,

$$
t_{i}=\ell_{i} \pm \sqrt{\ell_{i}^{2}-2 w_{i}}
$$

and the smaller root is the running time of the job. (This can be seen since the larger root is greater than $\ell$, the interval length.)

The total time taken by all $n-1$ nonoverlapping jobs when scheduled optimally is therefore

$$
\sum_{i=1}^{n-1} \ell_{i}-\sum_{i=1}^{n-1} \sqrt{\ell_{i}^{2}-2 w_{i}}=\sum_{i=1}^{n-1} \ell_{i}-\sum_{i=1}^{n-1} \sqrt{x_{i}} .
$$


(a)

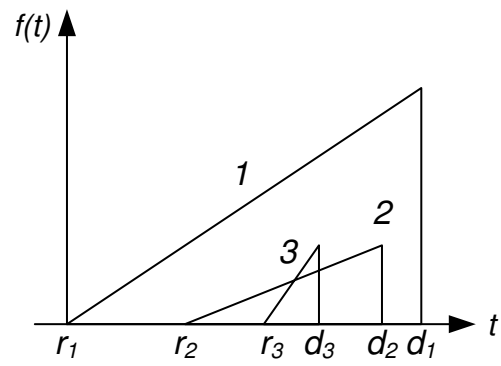

(b)

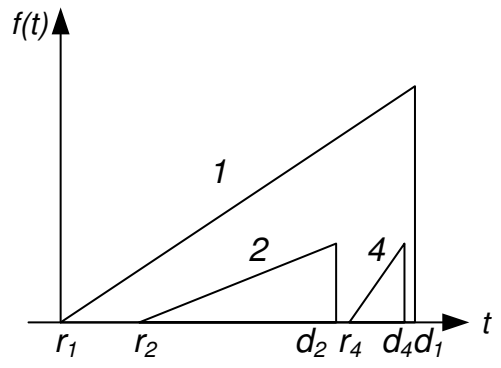

Figure 2: (a) Case 1: job 1 is run at time $r_{2}=3$. Then job 3 arrives. Either job 2 or 3 is forced to miss its due date. (b) Case 2: job 2 is run at time $r_{2}=2$. Then job 4 arrives. Either job 1, 2, or 4 is forced to miss its due date.

We now construct the nonlazy job $n$. As described earlier $r_{n}=0$ and $d_{n}=d_{n-1}$. We set work $w_{n}=I$. There is a feasible solution for this scheduling problem if and only if

$$
d_{n} \geq w_{n}+\sum_{i=1}^{n-1} \ell_{i}-\sum_{i=1}^{n-1} \sqrt{x_{i}} .
$$

This is the case, as long as $I \leq \sum_{i=1}^{n-1} \sqrt{x_{i}}$, since by construction, $d_{n}=d_{n-1}=\sum_{i=1}^{n-1} \ell_{i}$. Thus, an arbitrary instance of the sum-of-square-roots problem can be reduced to an instance of procrastination scheduling, implying the numerical difficulty of procrastination scheduling.

\section{Online Algorithms}

This section considers the online procrastination scheduling problem. In the online problem, jobs $1 \ldots n$ arrive over time. Job $j$ is known to the scheduler only at the release time $r_{j}$, at which point the scheduler also learns the values of $w_{j}$ and $d_{j}$. We first show that it is difficult for an online scheduler to find feasible schedules. Next we search for online algorithms that generate small, ideally constant, max-stretch. We show that traditional scheduling policies such as EDD, SRPT, and FIFO, have large, typically unbounded, max-stretch. We next consider the scheduling policy Largest-Stretch-So-Far (LSSF), which executes the job in the system currently having the largest interval stretch. This policy formalizes the "hit-the-highest-nail" scheduling policy, that is, execute the task in the system that most crucially requires attention. More precisely, in the LSSF scheduling policy, we run the job in the system that has incurred the largest interval stretch so far, that is, at time $t$ we execute the job $j$ that maximizes $\left(t-r_{j}\right) /\left(d_{j}-r_{j}\right)$. We show that, remarkably, LSSF also has unbounded max-stretch. We conclude this section by exhibiting the scheduling algorithm THRASHING, whose max-stretch is within a constant factor of optimal and then give a generalization to non-linear speed functions. One consequence of this last result is that good online max-interval-stretch bounds are achievable even when the procrastinator's maximum processing speed is at most a constant factor faster than a nonprocrastinator's speed.

\section{Basic Results}

We first show that any online algorithm can be forced to miss due dates, even when the scheduling instance is feasible. A job $j$ has slack if the work, $w_{j}$, associated with it is less than the area between $r_{j}$ and $d_{j}$, i.e., $w_{j}<\left(d_{j}-r_{j}\right)^{2} / 2$.

Theorem 3 For any online algorithm, there is a feasible job stream on which that algorithm misses due dates. 
Proof. We show that regardless of the online scheduling decisions, the adversary can force the algorithm to miss due dates by maliciously selecting future jobs. The adversary first sends jobs 1 and 2 , where $r_{1}<r_{2}$ and $d_{2}<d_{1}$. Both jobs 1 and 2 have some slack and the set $\{1,2\}$ is feasible. At time $r_{2}$ there are two cases:

1. Job 1 is serviced at time $r_{2}$. Then the adversary places a job 3 with $r_{2}<r_{3}<d_{3}<d_{2}$. Job 3 is designed so that the entire interval $\left[r_{2}, d_{2}\right]$ is required to complete jobs 2 and 3 by their due dates. Since the online algorithm works partially on job 1 during this interval, either job 2 or 3 misses its due date; see Figure 2 (a).

2. Job 2 is serviced at time $r_{2}$. The adversary places a job 4 with $r_{4}>d_{2}$ and $d_{4}<d_{1}$. Job 4 is designed so that all the time between $r_{2}$ and $d_{1}$ is required to complete jobs 1,2 , and 4 by their due dates. However, as job 2 has some slack we know that by Theorem 1 that the optimal strategy is to run 1 at time $r_{2}$ and that this strategy is unique. Therefore, by running 2 at time $r_{2}$ the algorithm misses at least one of the due dates; see Figure 2 (b).

Observe that, as stated, this example has job parameters that may be irrational (because of square roots). In fact, we can round job parameters so that all are rational and the input size (number of bits) is polynomial in $n$.

By repeating this construction, the adversary can force the algorithm to miss an arbitrarily large number of due dates. Thus, Theorem 3 explains why procrastinators may have a harder time juggling online tasks than non-procrastinators.

We now show that most traditional scheduling policies for non-procrastinators do not work well for procrastinators. The following theorem gives the performance of First-In-First-Out (FIFO), Earliest-DueDate (EDD), and Shortest-Remaining-Processing-Time (SRPT).

Theorem 4 There exist feasible scheduling instances of a constant number of jobs for which the maxstretch of the First-In-First-Out (FIFO) and Earliest-Due-Date (EDD) scheduling policies can be arbitrarily large. There exist feasible scheduling instances of n jobs for which the Shortest-Remaining-Processing-Time (SRPT) scheduling policy achieves a max-stretch of $\Theta(\sqrt{n})$.

Proof. There is a bad example for FIFO consisting of only two jobs. Let $r_{1}<r_{2}<d_{2}<d_{1}$. Set $w_{1}$ and $w_{2}$ so that optimal schedule is to execute job 2 to completion as soon as it arrives, and then finish job 1 . In FIFO, job 2 will not start work until job 1 has completed and will finish late. The interval stretch of job 2 can be made arbitrarily large by decreasing $w_{2}$ and $d_{2}-r_{2}$ or by increasing $d_{1}$ and $w_{1}$.

There is a bad example for EDD consisting of only three jobs. As before, let $r_{1}<r_{2}<d_{2}<d_{1}$. In EDD, job 2 is executed starting at its arrival time $r_{2}$ because this job has the earliest deadline. By the proof of Theorem 1 job 1 can be made finish its work after its due date. Now set a third job so that $r_{3}=d_{1}$ and $d_{3}-r_{3}$ is small compared to the lateness of job 1 . The interval stretch of job 3 can be made arbitrarily large by decreasing $d_{3}-r_{3}$ or by increasing the lateness of job 1 .

There is a bad example for SRPT consisting of $n$ jobs. All jobs are released at time 0 . Give job 1 the largest amount of work: $w_{1}=1$. Give all other jobs $w_{2}=w_{3}=\cdots=w_{n}=1 / 2$. Set $d_{1}$ so that job 1 must be executed as soon as it arrives in order not to be late, i.e., $d_{1}=2$. Give all other jobs later deadlines: $d_{2}=d_{3}=\cdots=d_{n}=\sqrt{n}+2$. In the optimal schedule, job 1 is executed first and the remaining jobs are executed in any order. In contrast, in SRPT, jobs $2 \ldots n$ are executed before job 1 . One job will be completed at time 1 , the next at time $\sqrt{2}$, the next at time $\sqrt{3}$, and the last at time $\sqrt{n-1}$. (A calculation similar to this is explained in greater detail in the next section.) Only after all other jobs complete does job 1 complete, giving it an interval stretch of $\Omega(\sqrt{n})$. 

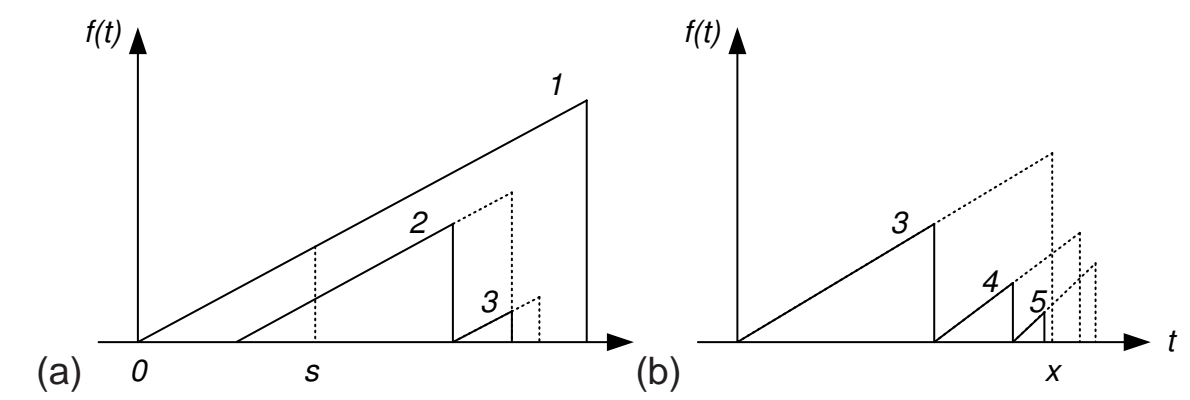

Figure 3: (a) Job 2 starts work at $s$ and completes after its due date. Job 3 starts work at its due date and finishes with a stretch of $\sqrt{2}$ (b) A stream of jobs with increasing stretch. The stretch of job 4 is $\sqrt{2}$ when it starts work at time $x$ and $\sqrt{3}$ when it finishes.

\section{Hitting the Highest Nail Does Not Work}

A common scheduling strategy among procrastinators is "hit-the-highest-nail," that is, execute the job that is farthest behind. Since the objective is to minimize the max-stretch, "hitting-the-highest-nail" translates to running the job that has the largest interval stretch. We call this strategy Largest-Stretch-So-Far (LSSF). More precisely, in the LSSF scheduling policy, we run the job in the system that has incurred the largest interval stretch so far, that is, at time $t$ we execute the job $j$ that maximizes $\left(t-r_{j}\right) /\left(d_{j}-r_{j}\right)$. Thus, the algorithm might execute a job $i$, but switch to a smaller job $j$ that arrived after $i$, once $j$ 's interval-stretchso-far surpasses that of $i$ 's.

Remarkably, even for feasible scheduling instances, LSSF may schedule jobs to have unbounded maxstretch. Below we exhibit such an adversarial scheduling instance that confounds LSSF. For simplicity, we describe a scheduling instance where job parameters may be irrational because of square roots. We then show how to round the job parameters so that all are rational.

Our bad instance consists of $n$ jobs, indexed by increasing arrival time. We ensure that jobs $2 \ldots n$ have no slack, that is,

$$
w_{j}=\frac{\left(d_{j}-r_{j}\right)^{2}}{2} \quad(2 \leq j \leq n) .
$$

Thus, in order for job $j(2 \leq j \leq n)$ to complete by its deadline, job $j$ must be executed without pause during its entire interval. In contrast, job 1 does have slack.

We arrange jobs 1-3 so that in LSSF, job 3 does not begin executing until after its due date $d_{3}$. To do so, we assign intervals for jobs 1 and 2 so that $r_{2}>r_{1}, d_{2}<d_{1}$, and $d_{1}-r_{1}=O(1)$. Thus, in the LSSF schedule, job 1 works uninterrupted until some point in job 2's interval when job 2 has the largest stretchso-far and so begins executing. Since job 2 has no slack, it finishes late, after its deadline $d_{2}$. We place job 3 so its release time is job 2's deadline and its deadline is job 2's completion time, i.e., $r_{3}=d_{2}$ and $d_{3}=C_{2}$. In LSSF, job 3 does not start until its due date, $d_{3}$, and then works uninterrupted until it completes for an interval stretch of $s_{3}=\sqrt{2}$; see Figure 3 (a).

We now assign jobs $4 \ldots n$ as follows; see Figure 3 (b). Each job $j$ has release time

$$
r_{j}=d_{j-1} \quad(3 \leq j \leq n) .
$$

Moreover, in LSSF we assign $d_{j}$ so that job $j$ has a stretch-so-far at time $C_{j-1}$ of

$$
\frac{C_{j-1}-r_{j}}{d_{j}-r_{j}}=s_{j-1}=\frac{C_{j-1}-r_{j-1}}{d_{j-1}-r_{j-1}} \quad(4 \leq j \leq n) .
$$

In the following we show that in LSSF, $s_{n}=\Theta(\sqrt{n})$. In contrast, in OPT, all jobs finish before their deadlines: jobs $2 \ldots n$ run in their intervals and job 1 begins before job 2 arrives and completes after job $n$ completes. 
We now analyze the performance of LSSF on these $n$ jobs.

Theorem 5 There exist feasible scheduling instances of njobs for which the scheduling policy LSSF achieves a max-stretch of $\Theta(\sqrt{n})$.

Proof. We analyze the performance of LSSF on the schedule instance given above. We derive a recurrence for the stretch $s_{j}$ as a function of $s_{j-1}$. Then we solve the recurrence, showing that $s_{n}=\Theta(\sqrt{n})$.

Define interval $I_{j}=d_{j}-r_{j}$. Recall that in LSSF, job $j$ only begins executing at time $C_{j-1}=r_{j}+s_{j-1} I_{j}$ because, from (4) and (5), while job $j-1$ is in the system, its stretch-so-far is larger than that of job $j$ 's.

We now determine the time $x_{j}$ that job $j$ spends running. By Equations (3) and (5), we have

$$
w_{j}=\frac{I_{j}^{2}}{2}=s_{j-1} I_{j} x_{j}+\frac{x_{j}^{2}}{2} \quad(j \geq 4) .
$$

Solving for $x_{j}$ and taking the positive root, we obtain

$$
x_{j}=-s_{j-1} I_{j}+I_{j} \sqrt{1+s_{j-1}^{2}} \quad(j \geq 4) .
$$

Thus, the stretch is

$$
s_{j}=\frac{C_{j-1}+x_{j}-r_{j}}{I_{j}} \quad(j \geq 4) .
$$

From (5), the previous equation simplifies to

$$
s_{j}=s_{j-1}+\frac{x_{j}}{I_{j}} \quad(j \geq 4) .
$$

Finally, from (6) we substitute for $x_{j}$, obtaining

$$
s_{j}=\sqrt{1+s_{j-1}^{2}} \quad(j \geq 4) .
$$

The solution to this recurrence is

$$
s_{j}=\sqrt{j-1} \quad(j \geq 3),
$$

meaning that the max-stretch is $s_{n}=\sqrt{n-1}$.

We now show how big job 1's interval has to be for the entire scheduling instance to be feasible. We make a recurrence for the interval length $I_{j}$. By (5), we obtain

$$
I_{j}=I_{j-1}\left(\frac{C_{j-1}-r_{j}}{C_{j-1}-r_{j-1}}\right)=I_{j-1}\left(1+\frac{r_{j-1}-r_{j}}{C_{j-1}-r_{j-1}}\right) \quad(j \geq 4) .
$$

Finally, by Equations (4) and (7), we obtain

$$
I_{j}=I_{j-1}\left(1+\frac{1}{s_{j-1}}\right)=I_{j-1}\left(1-\frac{1}{\sqrt{j-2}}\right) \quad(j \geq 4) .
$$

Therefore, assuming w.l.o.g. that $I_{3}=1$, an upper bound on $I_{j}$ is

$$
I_{j}=\prod_{i=2}^{j-2}\left(1-\frac{1}{\sqrt{i}}\right) \leq e^{-\sqrt{j-3}} \quad(j \geq 3) .
$$


The sum of all intervals lengths is

$$
\sum_{j=3}^{n} I_{j}=\sum_{j=3}^{n} e^{-\sqrt{j-3}}=O(1) \quad(j \geq 3) .
$$

Consequently, it suffices to set $I_{1}=O(1)$ and $w_{1}=O(1)$ to obtain a feasible schedule.

This particular example has job parameters that may be irrational (because of square roots). In fact, we can come up with another scheduling instance so that the input size (number of bits necessary to describe the scheduling instance) is polynomial in $n$. The idea is to round job parameters so that all are rational. We round the interval length $d_{j}-r_{j}$ of job $j$ up to a rational number and round the work $w_{j}$ down to a rational number. We make both $r_{j}$ and $d_{j}$ rational and retain Equation (4). We make the equality in Equations (3) and (5) only approximate, that is, for arbitrarily small nonnegative $\varepsilon_{j}$ and $\varepsilon_{j}^{\prime}$,

$$
\begin{array}{cc}
w_{j}+\varepsilon_{j}=\left(d_{j}-r_{j}\right)^{2} / 2 & (2 \leq j \leq n) \\
\frac{C_{j-1}-r_{j}}{d_{j}-r_{j}}+\varepsilon_{j}^{\prime}=s_{j-1} & (4 \leq j \leq n) .
\end{array}
$$

The analysis for Theorem 5 carries over.

\section{$\Theta(1)$-Competitive Online Algorithm for Max-Stretch}

We now exhibit the strategy THRASHING, which bounds the interval stretch of each job by 4 . The THRASHING strategy models the extreme case of a procrastinator who does not work on any job until it has already passed its due date. More formally, in the this strategy no job is executed until it has a stretch of at least 2. Among all such jobs, the procrastinator executes the job that arrived latest.

Before proceeding, we explain our choice of terminology. An operating system is said to 'thrash' when it begins running inefficiently because it spends too much time context switching. 'Thrashing' is now also commonly used among computer scientists to describe their own behavior when they have too many jobs to finish. The name is applied here because the procrastinator appears to be thrashing. Each time a more recent job has too large an interval stretch, the procrastinator abandons the current job and executes the more recent job.

We begin by proving the following simple lemma:

Lemma 6 Consider a feasible set of jobs $1, \ldots, m$ and consider times $r$ and $d$, where all $r_{j} \geq r$ and $d_{j} \leq d$. Let $\alpha-D L Y$ be any scheduling policy that only schedules work from jobs having stretch at least $\alpha$, where $\alpha \geq 1$. The total amount of time required to run all jobs using $\alpha-D L Y$ is at most $(d-r) / \alpha$.

Proof. Because the set of jobs is feasible, there is some way to schedule each job within its interval and the total time spent working is at most $d-r$. Now consider running $\alpha$-DLY. For any given job $j$, the slowest that $j$ runs in $\alpha$-DLY is at least $\alpha$ times faster than $j$ runs in the feasible schedule. The lemma follows immediately.

Theorem 7 For any feasible set of jobs, THRASHING bounds the interval stretch of every job by 4.

Proof. The proof is by contradiction. Define the extended due date $\tilde{d}_{j}$ of job $j$ to be the time that $j$ must complete by to guarantee an interval stretch of 4 , that is, $\tilde{d}_{j}=4\left(d_{j}-r_{j}\right)+r_{j}$. Consider some job $j$ that does not meet its extended due date. For simplicity and without loss of generality, we normalize time so that $r_{j}=0$ and $d_{j}=1$. Job $j$ cannot begin until time 2 and by assumption completes at some time $f>4$. 
By Lemma 6 the total amount of time spent working on all jobs (including $j$ ) whose intervals are entirely contained within $[0,4]$ is at most $4 / 2=2$ units of time. Moreover, there can be no gaps in the schedule during the interval $[2, f]$ because otherwise $j$ would work during the gaps and finish earlier than time $f$. Finally, by the definition of THRASHING, there can be no work scheduled during $[2, f]$ on jobs having release dates before 0 because $j$ has higher priority. Thus, $f$ cannot be greater than 4 and we obtain a contradiction.

It may, of course, be unrealistically optimistic to give the online procrastinator the power to run arbitrarily fast. However, it follows from Theorem 7 that THRASHING never runs any job $j$ faster than $4 f_{j}\left(d_{j}\right)$. In fact, the proof of Theorem 7 indicates that we can reduce this upper bound still further to $2 f_{j}\left(d_{j}\right)$ without increasing the max-stretch; we need only modify the speed functions so that the maximum job speed for job $j$ is limited to $2 f_{j}\left(d_{j}\right)$.

\section{Conclusions}

The first sentence of the conclusion, which summarizes the paper, is being written just a few hours before the deadline. As we were writing this paper, we were struck by the wealth of open problems in this area. For example, what is the right way to resolve the computational and numerical issues associated with linear and other speed functions? The scheduling problem (even in the offline case) becomes even more complex with speed functions that may be nonzero at jobs' release times. (This is because LRTB fails, and the optimal schedule seems to depend on the workload as well as on the slopes of the speed functions.) For our online algorithm we did not try to optimize the constant in the online competitive ratio fully; what is the smallest that we can make this constant, especially where the speed functions are sublinear?

We have also considered piecewise-constant speed functions and have linear programming solutions for several variants of the problem. The LP has constraints for each time interval $\left[t_{1}, t_{2}\right]$ in which the execution speeds of all jobs are constant. (Specifically, within $\left(t_{1}, t_{2}\right)$ there are no job release times or deadlines, and for each job $j$ the function of $f_{j}(t)$ is constant when $t \in\left[t_{1}, t_{2}\right]$.) There are many metrics we can optimize. For example, we can minimize or maximize the total amount of time working. Alternatively, we can introduce a notion of stress for the procrastinator and find the least stressful schedule.

Finally, what about other metrics, especially in models where some jobs may be left unexecuted? What about settings where job streams are executed on parallel processors?

It is now several hours later, just minutes before the deadline. We were searching for the ideal way to end the paper and circumstances have unfortunately provided the answer. A campus-wide power failure at Stony Brook has cut two hours from our last-minute working time and highlights the difficulties of online scheduling for procrastinators.

\section{Acknowledgments}

We are grateful to Esther Arkin, Nikhil Bansal, and Joseph Mitchell for many helpful discussions. We thank Nikhil Bansal for the LP solution for piecewise constant speed functions.

\section{References}

[1] S. Albers and H. Fujiwara. Energy-efficient algorithms for flow time minimization. In Proc. 23rd Annual Symposium on Theoretical Aspects of Computer Science (STACS), volume 3884 of Lecture Notes in Computer Science, pages 621-633, 2006.

[2] B. Alidaee and K. Womer. Scheduling with time dependent processing times: Review and extensions. Journal of Operational Research Society, 50:711-720, 1999. 
[3] E. M. Arkin, M. A. Bender, J. S. B. Mitchell, and S. S. Skiena. The lazy bureaucrat scheduling problem. In Proc. 6th Workshop on Discrete Algorithms WADS, pages 122-133, 1999.

[4] E. M. Arkin, M. A. Bender, J. S. B. Mitchell, and S. S. Skiena. The lazy bureaucrat scheduling problem. Information and Computation, 184(1):129-146, 2003.

[5] A. Bachman, A. Janiak, and M. Y. Kovalyov. Minimizing the total weighted completion time of deteriorating jobs. Information Processing Letters, 81(2):81-84, 2002.

[6] N. Bansal, T. Kimbrel, and K. Pruhs. Dynamic speed scaling to manage energy and temperature. In Proc. 45th Symposium on Foundations of Computer Science (FOCS), pages 520-529, 2004.

[7] N. Bansal and K. Pruhs. Speed scaling to manage temperature. In Proc. 22nd Annual Symposium on Theoretical Aspects of Computer Science (STACS), volume 3404 of Lecture Notes in Computer Science, pages 460-471, 2005.

[8] M. A. Bender, S. Chakrabarti, and S. Muthukrishnan. Flow and stretch metrics for scheduling continuous job streams. In Proceedings of the 9th Annual ACM-SIAM Symposium on Discrete Algorithms (SODA), pages 270-279, 1998.

[9] M. A. Bender and M. O. Rabin. Online scheduling of parallel programs on heterogeneous systems with applications to Cilk. Theory of Computing Systems Special Issue on SPAA00, 35:289-304, 2002.

[10] D. P. Bunde. Power-aware scheduling for makespan and flow. In Proc. 18th ACM Symposium on Parallelism in Algorithms and Architectures (SPAA), 2006. To appear.

[11] C. Chekuri and M. A. Bender. An efficient approximation algorithm for minimizing makespan on uniformly related machines. Journal of Algorithms, 41:212-224, 2001.

[12] F. A. Chudak and D. B. Shmoys. Approximation algorithms for precedence-constrained scheduling problems on parallel machines that run at different speeds. J. Algorithms, 30(2):323-343, 1999. An earlier version appears in SODA '97.

[13] E. Davis and J. M. Jaffe. Algorithms for scheduling tasks on unrelated processors. J. ACM, 28(4):721-736, 1981.

[14] E. D. Demaine, J. S. B. Mitchell, and J. O'Rourke. The open problems project. http://maven.smith.edu/ orourke/TOPP/, viewed February 13, 2005.

[15] D. Eppstein. Geometry junkyard, computational and recreational geometry pointers. http://www.ics.uci.edu/ eppstein/junkyard/open.html viewed April 6, 2007.

[16] L. Fleischer and M. Skutella. The quickest multicommodity flow problem. In Proc. 9th Integer Programming and Combinatorial Optimization (IPCO) Conference, volume 2337 of Lecture Notes in Computer Science, pages 36-53, 2002.

[17] L. Fleischer and M. Skutella. Minimum cost flows over time without intermediate storage. In Proc. 14th Annual ACM-SIAM Symposium on Discrete Algorithms (SODA), pages 66-75, 2003.

[18] S. Gawiejnowicz, W. Kurc, and L. Pankowska. A greedy approach for a time-dependent scheduling problem. LNCS, 2328:7986,2002

[19] S. Gawiejnowicz and L. Pankowska. Scheduling jobs with varying processing times. Information Processing Letters, 54(3):175-178, 12 May 1995.

[20] C. Hepner and C. Stein. Minimizing makespan for the lazy bureaucrat problem. In Proc. 8th Scandinavian Workshop on Algorithm Theory (SWAT), volume 2368 of Lecture Notes in Computer Science, pages 40-50, 2002.

[21] S. Irani and K. R. Pruhs. Algorithmic problems in power management. SIGACT News, 36(2):63-76, 2005.

[22] K. Jansen and L. Porkolab. Improved approximation schemes for scheduling unrelated parallel machines. In Proc. 31st Annual ACM Symposium on Theory of Computing, pages 408-417, 1999.

[23] D. Karger, C. Stein, and J. Wein. Scheduling algorithms. In M. J. Atallah, editor, Handbook of Algorithms and Theory of Computation. CRC Press, 1998.

[24] E. L. Lawler and J. Labetoulle. On preemptive scheduling of unrelated parallel processors by linear programming. J. ACM, 25(4):612-619, 1978

[25] A. Orda and R. Rom. Shortest-path and minimum-delay algorithms in networks with time-dependent edge-length. J. ACM, 37(3):607-625, 1990.

[26] J. O’Rourke. Advanced problem 6369. Amer. Math. Monthly, 1981.

[27] K. Pruhs, R. van Stee, and P. Uthaisombut. Speed scaling of tasks with precedence constraints. In Proc. 3rd Workshop on Approximation and Online Algorithms (WAOA), pages 307-319, 2005.

[28] E. Uysal-Biyikoglu, B. Prabhakar, and A. El Gamal. Energy-efficient packet transmission over a wireless link. IEEE/ACM Trans. Netw., 10(4):487-499, 2002. 